J. Phys. IV France 132 (2006) 177-183

(C) EDP Sciences, Les Ulis

DOI: $10.1051 /$ jp4:2006132034

\title{
Observation of growth during the MOVPE of III-nitrides
}

\author{
H. Hardtdegen ${ }^{1}$, N. Kaluza ${ }^{1}$, R. Steins ${ }^{1}$, Y.S. Cho ${ }^{1}$, R. Schmidt ${ }^{1}$, \\ Z. Sofer ${ }^{1}$ and J.-T. Zettler ${ }^{2}$ \\ ${ }^{1}$ Institute of Thin Films and Interfaces, Center of Nanoelectronic Systems for Information \\ Technology (CNI), Research Center Juelich, 52425 Juelich, Germany \\ 2 LayTec GmbH, Helmholtzstr. 13-14, 10587 Berlin, Germany
}

\begin{abstract}
This paper reports on how the observation of the morphology development and growth by in situ optical methods as well as the determination of substrate temperature can be employed to tailor the characteristics of GaN and to control growth in MOVPE (metalorganic vapor phase epitaxy). Furthermore for the first time a method will be demonstrated that allows the difficult determination of an alloy composition here $\left(\mathrm{Al}_{\mathrm{x}} \mathrm{Ga}_{1-\mathrm{x}}\right) \mathrm{N}$ - independent on the perfection and roughness of the developing layer.
\end{abstract}

\section{INTRODUCTION}

The growth of III-nitrides with high crystalline perfection is a great challenge since there is a lack of commercially available native substrates on which homoepitaxial growth could be performed. Usually substrates are employed such as sapphire which exhibit a lattice mismatch of $16 \%$ with respect to GaN leading to high dislocation densities of up to $10^{13} \mathrm{~cm}^{-2}$ in the deposited layers. An additional challenge especially for $\left(\mathrm{Al}_{\mathrm{x}} \mathrm{Ga}_{1-\mathrm{x}}\right) \mathrm{N}$ growth in MOVPE is the high deposition temperature and its exact control necessary to achieve good crystalline material. The determination and control of the substrate temperature is however not trivial if transparent substrates such as sapphire or $\mathrm{SiC}$ are employed. In order to deposit high quality material, GaN buffer growth strategies such as the two step growth procedure proposed by Amano et al. [1,2] were developed in order to reduce the dislocation density down to $10^{8} \mathrm{~cm}^{-2}-10^{9} \mathrm{~cm}^{-2}$. The crystalline quality of the resulting layer is determined by the morphology development from a nearly amorphous to a single crystalline layer. Therefore it is useful for material optimization to be able to directly observe the morphology development without intruding during growth and without destructing the sample. As soon as heterostructures are to be deposited, further layer characterization would be useful. Since a strong interdependence of growth parameters on composition is observed for the growth of $\left(\mathrm{Al}_{\mathrm{x}} \mathrm{Ga}_{1-\mathrm{x}}\right) \mathrm{N}$ alloys [3,4], the real-time determination of alloy composition in the reactor would be time-saving for tuning the material composition simultaneous to the optimization of growth.

In this paper we will report on how in situ optical methods can be used to measure the absolute surface temperature [5], to observe morphology development [6]. At last a new method will be discussed with which the alloy composition of $\left(\mathrm{Al}_{\mathrm{x}} \mathrm{Ga}_{1-\mathrm{x}}\right) \mathrm{N}$ layers can be determined.

\section{EXPERIMENTALS}

The experiments were carried out in a single two inch wafer horizontal reactor (AIX 200/4 RF-S, AIXTRON). Hydrogen and nitrogen were employed to transport the group III sources TMGa (trimethylgallium) and TMAl (trimethylaluminium) as well as the ammonia to the inductively heated graphite susceptor. The reactor is equipped with an in situ optical sensor (EpiR DA TT) which allowed the access through a strain-free quartz window to the substrate. By this sensor the simultaneous measurement of reflectance in the wavelength range between 250 and $825 \mathrm{~nm}$ and the determination of surface temperature using emissivity corrected pyrometry at $980 \mathrm{~nm}$ was carried out. The calibration 
of true surface temperature was performed using double side polished $450 \mu \mathrm{m}$ thick $6 \mathrm{H}-\mathrm{SiC}$ substrate as described in [5]. For growth experiments $450 \mu \mathrm{m}$ thick c-plane sapphire wafers with a misorientation of $0.2^{\circ}$ towards the a-plane were employed. The observation of the morphology development was carried out at a wavelength of $635 \mathrm{~nm}$. For the real-time determination of $\mathrm{Al}_{\mathrm{x}} \mathrm{Ga}_{1-\mathrm{x}} \mathrm{N}$ layer composition the reflectance transients at two sufficiently different wavelengths were chosen for the determination of the alloy composition. The $\mathrm{GaN}$ and $\mathrm{Al}_{\mathrm{x}} \mathrm{Ga}_{1-\mathrm{x}} \mathrm{N}$ layers were additionally characterized with well established ex situ, post growth methods: the morphology by atomic force microscopy, the composition by $\mathrm{x}$-ray diffraction and Rutherford backscattering spectroscopy and the thickness by white light interferometry.

\section{RESULTS AND DISCUSSION}

\subsection{Determination of true surface temperature for transparent substrates}

The surface temperature is determined by using pyrometry. It can however only be monitored if the measurement is corrected for emissivity changes. This is done by synchronously measuring the reflectance and reading the pyrometer at the same wavelength - typically $980 \mathrm{~nm}$. For absorbing substrates such as $\mathrm{Si}$ the temperature determination is directly possible. The accuracy is within $1^{\circ} \mathrm{C}$. For transparent substrates such as SiC and sapphire the procedure is different. Here the pyrometer "sees" through the wafer and reads the incandescence of the susceptor directly underneath the wafer. The offset of the wafer surface temperature to the underlying susceptor surface must be determined by a temperature calibration. Usually the calibration is done by determining the melting point of a metal or by determining the eutectic point of an alloy, a very well-know and defined temperature. A new method [5] is more easily accessible and uses the strong temperature dependence of the band gap of a semiconductor. Particularly suited for this determination is the semiconductor $\mathrm{SiC}$ due to its high temperature stability and its band gap position in the easy accessible visible spectral range. The onset of absorption is the sure indication that the band gap energy in the reflection measurement is approached. The absorption onset is best detected if the reflectance measured is normalized for practical purposes to the room temperature reflectance. The onset of the absorption correlates to the temperature in the reactor [7-9]. Fig. 1 displays the inferred reflectance spectra for an un-doped SiC wafer. The onset of absorption is clearly seen by a sharp falling edge in the spectra. With increasing substrate temperature the position of the falling edge moves to lower energies as the band gap decreases. Using a suitable fit function (straight lines in Fig. 1) the position of the absorption onset versus the absolute temperature was determined in a reactor in which the temperature calibration was carried out in the usual way. The respective absorption onset dependence (determined as described above) on absolute temperature is presented in Fig. 2 as a full line. Now, a gauging normal is established, the absorption onset is directly correlated to the absolute temperature. Using this reference measurement the surface temperature in our un-calibrated reactor can now be calibrated with an accuracy within $3^{\circ} \mathrm{C}$ just by repeating the above procedure and comparing the measured dependence to the reference measurement. The original measurement data as well as the fit (denoted as a dotted line) are also presented in Fig. 2. Assuming that sapphire wafers of equal thickness qualitatively show the same thermal behavior the calibration method can also be employed to determine the surface temperature of sapphire wafers during growth. Once calibrated in this manner the emissivity corrected pyrometer measures the correct wafer temperature for all growth runs on transparent substrates such as $\mathrm{SiC}$ and sapphire, provided that the wafer does not exhibit curvature or its surface is roughened.

For growth optimization it is important to be sure that the parameter that is being optimized does not intentionally change the surface temperature. In the past it has been shown that especially in CVD processes this can be the case $[10,11]$. The true temperature determination was therefore used to see which influence the ambient has on the surface temperature. To this end the ambient composition was varied from pure hydrogen to pure nitrogen. The experiment is of special interest for the comparison of In-alloy deposition since growth is preferentially performed in nitrogen ambient. All in all a strong decrease of surface temperature of $50^{\circ} \mathrm{C}$ is observed as the hydrogen ambient content decreases and the 


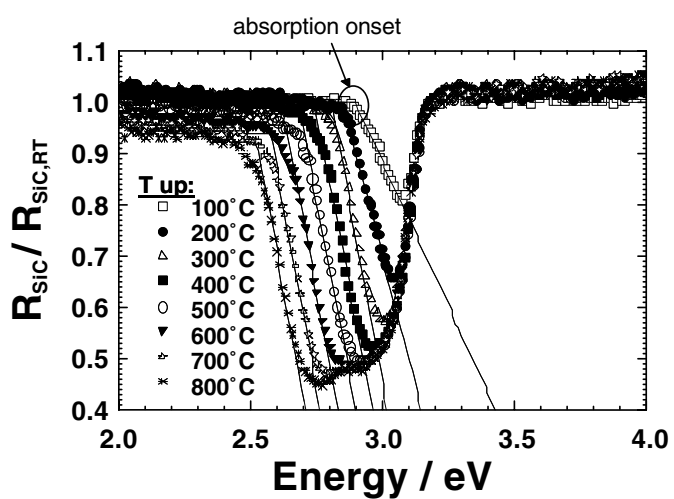

Figure 1. Normalized reflectance spectra of an undoped $\mathrm{SiC}$ wafer for a range of temperatures. The straight lines represent the fit function used to determine the onset of the absorption.

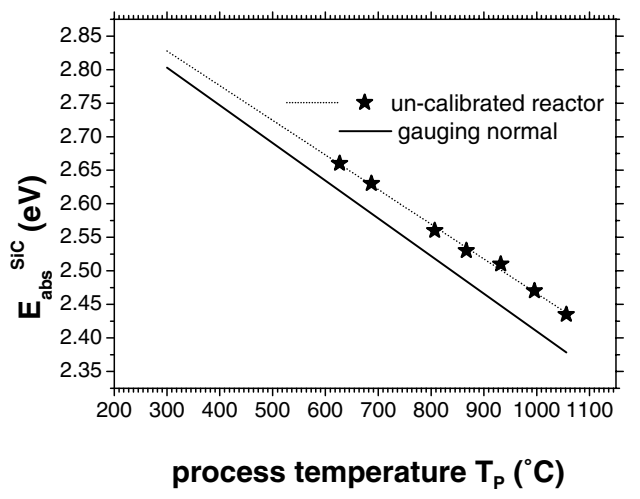

Figure 2. Correlation between absorption onset and absolute temperature: full line gauging normal; dotted line is a fit for the measurements in the un-calibrated reactor.

ambient gradually changes to nitrogen. Further details to these experiments can be found in reference [12]. This finding demonstrates the importance of true surface temperature determination. The method allows the absolute comparison of the growth temperature for different reactors.

\subsection{Use of reflectance transients for the optimization of nitride growth}

The morphology development in nitride growth lays the foundation of the bulk layer characteristics. According to the two step growth process [1,2], first an about $25 \mathrm{~nm}$ thick predominantly amorphous $\mathrm{GaN}$ layer is deposited at temperatures of around $500^{\circ} \mathrm{C}$. This layer is annealed at temperatures around $1000^{\circ} \mathrm{C}$ during which the layer is partially etched and partially crystallizes. Then in a second step deposition is carried out at temperatures close to the annealing temperature. At first the crystalline nuclei grow gradually. Only after they coalesce growth changes from the three dimensional to the two dimensional growth mode. At the boundaries between the coalescing crystallites dislocations form. In order to produce layers with a low dislocation density it is therefore advantageous to allow the nuclei to enlarge before they coalesce. After the coalescence phase is completed, only a small influence can be taken on the layer quality by the growth parameters. Therefore the morphology development needs to be observed closely in this first phase of growth. At different stages during growth the surface morphology was studied by stopping growth and observing the morphology by atomic force microscopy (AFM). Fig. 3 presents the surface morphology observed after (a) low temperature nucleation layer is deposited, (b) after annealing this $25 \mathrm{~nm}$ thick layer in hydrogen ambient, (c) after resuming growth during the coalescence phase and (d) in the two-dimensional layer by layer growth mode well described for example by Figge et al. [13]. Figure 3 also contains the recorded reflectance spectrum in which the different growth stages are denoted. Basically, the intensity development of the Fabry-Perot oscillations is a direct reflection of the surface morphology development. An overall decreasing or increasing roughness on the nanometer scale (the scale much smaller than the transient's wavelength) will tend to increase or decrease the overall average intensity of the oscillations This effect is demonstrated in figure $4 \mathrm{a}$. The roughness can best be described by the effective medium theory. An overall dampening of the amplitude of the oscillations at the same average intensity can only be due to optical thickness fluctuations (either thickness or refractive index fluctuations) on the micrometer scale which are averaged over the reflectance spot size on the sample) where beams with slightly different oscillation periods interfere (incoherent or coherent superposition of waves). This effect is presented in figure 4c. For comparison the reflectance transient for "ideal" $\mathrm{GaN} / \mathrm{sapphire}$ is presented in figure $4 \mathrm{~b}$. There are indications that refractive index fluctuations are the 

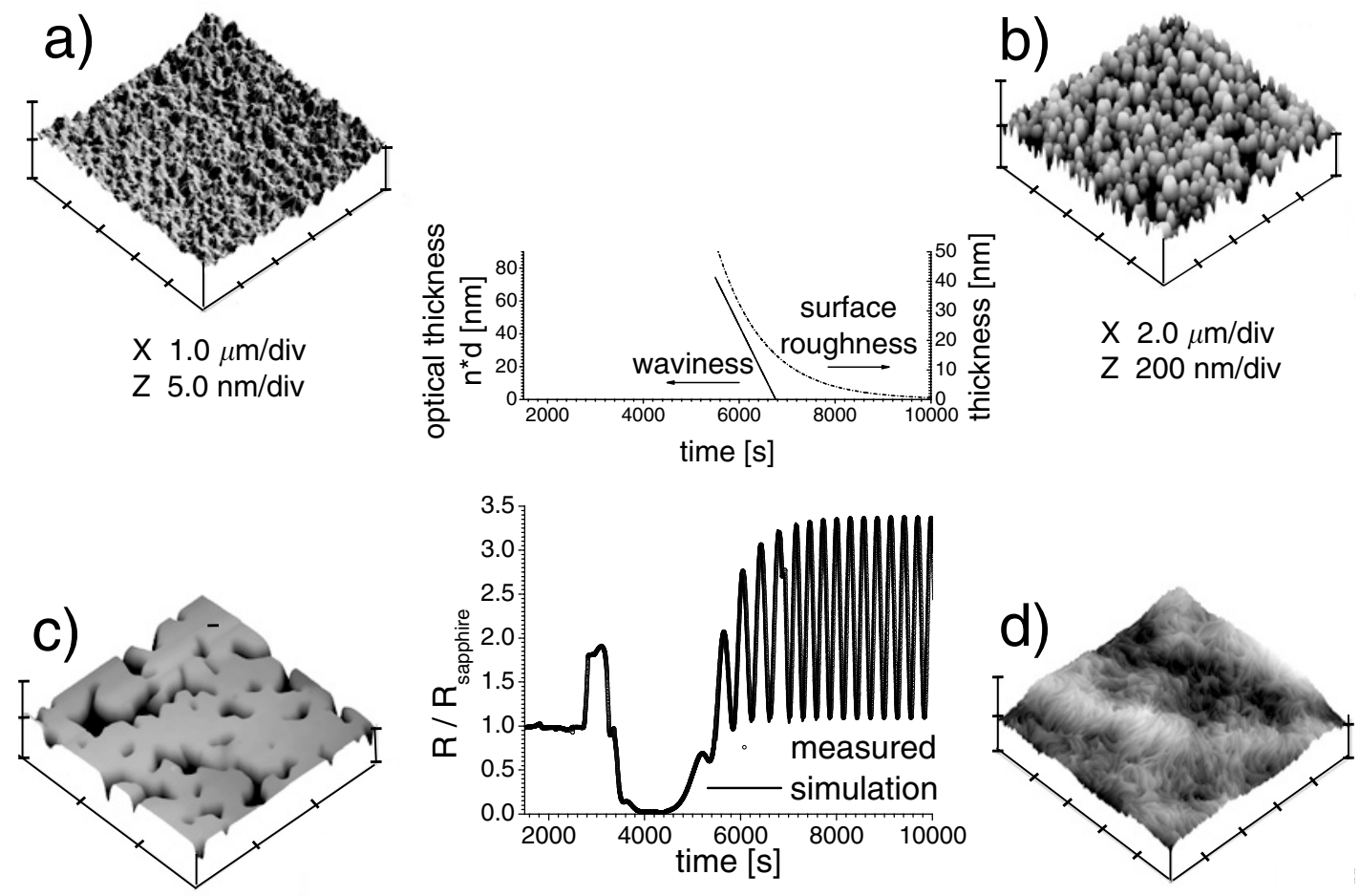

X $5.0 \mu \mathrm{m} / \mathrm{div}$

Z $500 \mathrm{~nm} / \mathrm{div}$

$X 2.0 \mu \mathrm{m} / \mathrm{div}$

Z $5.0 \mathrm{~nm} / \mathrm{div}$

Figure 3. Reflectance transient recorded at $600 \mathrm{~nm}$. The stages of growth are denoted a) after nucleation, b) after annealing, c) during coalescence and d) in the two-dimensional growth regime. The insets show the respective atomic force microscopy pictures. The well-matching simulation is also presented (solid line). The upper diagram shows the used roughness developments for the simulation.
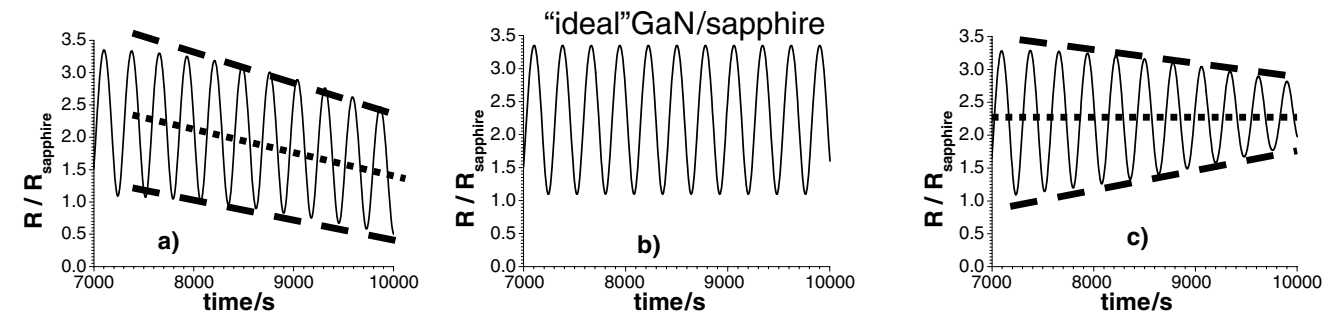

Figure 4. Effect on roughness on the reflectance transient for a GaN/sapphire layer growth: a) for increasing roughness on the nanometer scale; b) for ideal growth without roughness and c) for optical waviness on the micrometer scale.

main cause for the dampening, however further investigations are necessary to prove this assumption. Using this information the reflectance signal can be interpreted.

As can be seen in Fig 3a the low temperature nucleation layer is quite smooth, the reflectance signal therefore increases with thickness as the first oscillation develops. Then growth is stopped, the temperature increased and the layer annealed. Etching sets in and the reflectance signal drops, the layer becomes rough due to etching in the hydrogen ambient. At a certain point the reflectance increases as the smooth substrate 
surface partly is uncovered. The crystallites at this point are shown in Fig. 3b. Then growth is resumed. A smoothening of the layers occurs as the crystallites coalesce (Fig. 3c). The roughness decreases on the nanometer scale as the nuclei grow larger and coalesce. The overall average intensity of the reflectance increases. By using both scales for roughness development it becomes possible to model the growth transient [6] - as shown in Fig.3 - and then to improve growth strategies efficiently.

\subsection{Determination of $\mathrm{Al}_{\mathbf{X}} \mathrm{Ga}_{1-\mathrm{x}} \mathrm{N}$ composition}

In the past it has been shown that in situ optical methods are extremely useful during MOVPE growth for the fast and non-destructive determination of conventional III/V-alloy composition such as in the $\mathrm{AlAs} / \mathrm{GaAs}$ material system [10]. The reflectance signal (intensity) is dependent on the extinction coefficient and the refractive index. These values are characteristic of a material and therefore composition dependent. Since the change in dispersion coefficient and refractive index due to the deposition of the alloy both effect the transient in a similar way as layer roughness and waviness, the composition determination with the conventional method can only be applied for extremely smooth layers. The effect of an alloy on the reflectance transient is presented in figure 5 . In contrast to conventional III/ $\mathrm{V}$ 's, the growth perfection of nitrides is by far not so high and $\mathrm{Al}_{\mathrm{x}} \mathrm{Ga}_{1-\mathrm{x}} \mathrm{N}$ based layers are very often not completely smooth. The task of real-time and accurate measurement of composition for nitride alloys is therefore very challenging. Here the layer roughness may lead to errors of up to $100 \%$ in the composition when the determination is carried out by using the refractive indices and the extinction coefficient. Fig. 6 shows this calculated dependence for different $\mathrm{Al}_{\mathrm{x}} \mathrm{Ga}_{1-\mathrm{x}} \mathrm{N}$ concentrations.

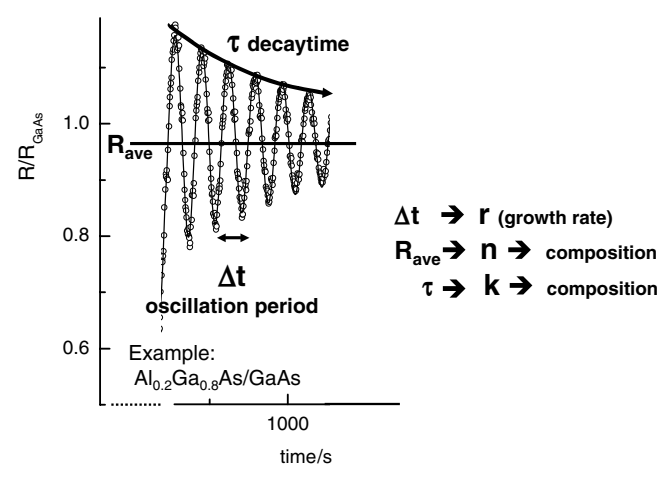

Figure 5. Influence of alloy growth on reflectance transient. The decay due to a material related change in dispersion coefficient has a similar effect as optical waviness, the material related change in refractive index has a similar effect on the transient as roughness on the nanometer scale.

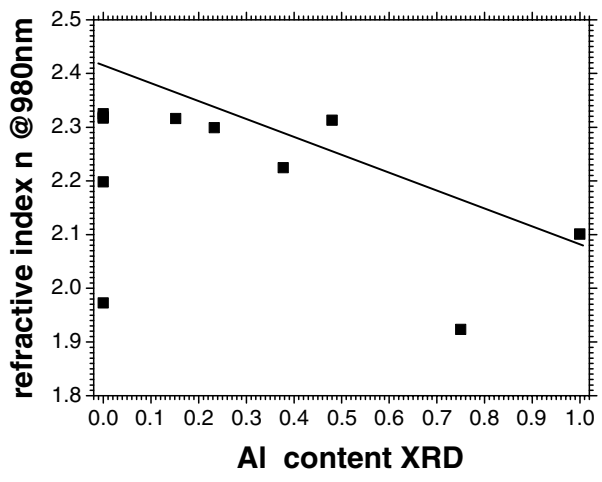

Figure 6. "Refractive index" determined at $980 \mathrm{~nm}$ for different $\mathrm{Al}_{\mathrm{x}} \mathrm{Ga}_{1-\mathrm{x}} \mathrm{N}$ layers on sapphire without taking roughness into account. A strong scatter of data is observed.

The refractive indices follow the expected trend: the higher the Al-content, the lower the refractive index. It is very clear that the data scatter is enormous. This is most probably due to surface roughness. The influence of surface roughness can be overcome if the refractive index ratio is calculated from transients recorded at two sufficiently different wavelengths instead of using only one refractive index. The wavelengths chosen were $450 \mathrm{~nm}$ in the UV range and the $980 \mathrm{~nm}$ for the IR range. In Figure 7 the dependence of the refractive index ratios is presented as a function of the $\mathrm{Al}_{\mathrm{x}} \mathrm{Ga}_{1-\mathrm{x}} \mathrm{N}$ compositions determined by X-ray diffraction (XRD). A great improvement in composition determination is achieved. A deviation of $\pm 5 \%$ instead of the over $100 \%$ is found. If an even lower wavelength of $322 \mathrm{~nm}$ is used for the high Al-content material with compositions above $40 \%$, where the wavelength is still quite far 


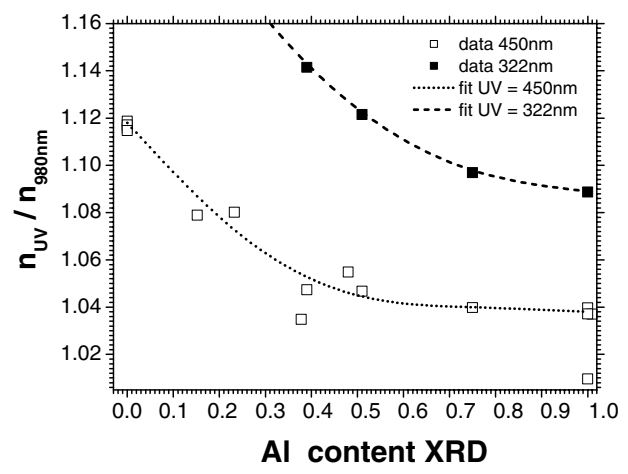

Figure 7. Determination of composition using the new dispersion approach taking into account surface roughness a great improvement of accuracy is achieved.

away from the onset of absorption, the fit to the data is even more precise and the error is smaller. This new method allows a fast first determination of the composition with a reasonable error bar.

\section{SUMMARY AND CONCLUSIONS}

The potential of in situ optical methods for the MOVPE growth of group III-nitrides was demonstrated. First the surface temperature for transparent substrates was measured with emissivity corrected pyrometry. A special temperature calibration method was used to determine the absolute temperature. This method employs the band gap energy dependence of un-doped $\mathrm{SiC}$ on temperature. With this method it was demonstrated that especially the carrier gas - in other words the hydrogen to nitrogen ratio unintentionally has a big influence on surface temperature. Furthermore the reflectance was recorded and the morphology development during growth was observed. An in situ optical model was introduced with which the reflectance transient was related to the morphology development. At last a new method was presented which allows the in situ determination of the Al-content in $\mathrm{Al}_{\mathrm{x}} \mathrm{Ga}_{1-\mathrm{x}} \mathrm{N}$ layers independent on the surface roughness. In spite of the time-consuming adjustment of several different growth parameters in order to obtain the chosen composition, the method has a reasonable accuracy so that at least the Al-content can be determined in real-time.

\section{Acknowledgments}

The authors would like to thank K. Wirtz for invaluable technical support. One of the authors, Z. Sofer, on leave from the Institute of Chemical Technology, Prague, Czech Republic, was supported by the EU program Leonardo da Vinci, project no. CZ/04/A/PL-134203 and the grant agency of Czech Republic, grant no. 104/03/0387. LayTec was supported by the "Arbeitsgemeinschaft industrieller Forschungsvereinigungen" in the PROINNO project no. KF 0445701 KFK2.

\section{References}

[1] H. Amano, N. Sawaki, I. Akasaki, Y. Toyoda, Appl. Phys. Lett. 48 (1986) 353-355.

[2] S. Nakamura, Jpn. J. Appl. Phys. 30 (1997) L1705-L1707.

[3] S.C. Choi, J.-H. Kim, J.Y. Choi, K.J. Lee, K.Y. Lim, G.M. Yang, J. Appl. Phys. 87 (2000) 172-176.

[4] T.G. Mihopoulos, V. Gupta, K.F. Jensen, J. Crystal Growth 195 (1998) 733-739.

[5] R. Steins, N. Kaluza, H. Hardtdegen, M. Zorn, K. Haberland, J.-T. Zettler, J. Crystal Growth 272 (2004) 81-86. 
[6] H. Hardtdegen, M. Zorn, J.-T. Zettler, "Benefits of optical in situ measurements for the MOVPE of compound semiconductor layers and device structures", Proceedings of the $12^{\text {th }}$ International Workshop on the Physics of Semiconductor Devices Chennai, India Dec. xx - yy 2003, eds. K.N. Bhat and A. DasGupta, Narosa Publishing House, New Delhi, (2004) 83-88.

[7] R.S. Balmer, T. Martin, J. Crystal Growth 248 (2003) 216-221.

[8] S.R. Johnson, C. Lavoie, T. Tiedje, J.A. Mackenzie, J. Vac. Sci. Technol. B. 11 (1993) 1007-1010.

[9] P.J. Timanns, J. Appl. Phys. 72 (1992) 660-670.

[10] K. Haberland, A. Kaluza, M. Zorn, M. Pristovsek, H. Hardtdegen, M. Weyers, J.-T. Zettler, W. Richter, J. Crystal Growth 240 (2002) 87-97.

[11] M. Dauelsberg, H. Hardtdegen, L. Kadinski, A. Kaluza, P. Kaufmann, J. Crystal Growth 223 (2001) 21-28.

[12] H. Hardtdegen, N. Kaluza, R. Steins, Y.S. Cho, Z. Sofer, M. Zorn, K. Haberland, J.-T. Zettler, to be published in Phys. Stat. Sol. (b).

[13] S. Figge, T. Böttcher, S. Einfeldt, D. Hommel, J. Crystal Growth 221 (2002) 262-266. 